\title{
Study on the frame load spectrum combining mileage and percentile extrapolation
}

\author{
Qingchun Song ${ }^{\mathrm{a}}$, Zhenghe Song and Yuefeng Du \\ Beijing Key Laboratory of Optimized Design for Modern Agricultural Equipment, College of \\ Engineering, CAU,Beijing, 10083, China
}

\begin{abstract}
In order to get the load spectrum that reflects the actual operation condition of agricultural machinery, this paper proposes to compile the load spectrum of frame by combining the method of load mileage extrapolation and percentile extrapolation. In this paper, the research object was carried out in the frame of vegetable tool carrier to study load spectrum and fatigue life. The finite element analysis of the frame is carried out, and a set of stress load test system is established based on the analysis results, and the stress time course is obtained under typical working conditions. The time domain signal is converted into frequency domain signal by rainflow counting method, and frequency statistics are carried out. According to the "From-to" matrix, the "Mean based ellipse" kernel function is used for non-parametric rainflow matrix extrapolation, considering the influence of different drivers in the actual operation, the load of three conditions is extrapolated by percentile. According to wave center method, developing eight two-dimensional load spectrum of the vegetable tool carrier frame and onedimensional load spectrum that reflects of actual loading situation, which provides a reference for the compilation of load spectrum in agricultural machinery.
\end{abstract}

\section{Introduction}

In the development stage of agricultural machinery products, the fatigue strength of structural parts should be investigated and the fatigue life should be evaluated. Dynamic loads exist in the use of mechanical structures, which will cause fatigue damage to components. The evaluation of fatigue damage is a very practical research content [1-2]. Load spectrum is the basis of component fatigue analysis, so it is particularly important to compile the load spectrum reflecting the actual working conditions [3]. Based on vegetable field machinery frame as the research object, combined with finite element analysis results to select relevant measuring point, testing load data through the establishment of load testing system and compiling the load spectrum, the fatigue life prediction and damage analysis was carried out on the frame. The structure design life is so long that it is difficult to obtain the load course in the service life through general test, so it is necessary to extrapolate the load course. Rodzewicz[4] integrated the measured data and extrapolated it to accurately predict the long-term load of glider. Socie[5] used the non-parametric estimation of rain flow matrix expansion method and applies it to practice, proving the effectiveness of the method. Drebler et al. [6] proposed a nonparametric extrapolation method to carry out loading process extrapolation by introducing kernel

\footnotetext{
${ }^{\mathrm{a}}$ Corresponding author : cauqingchun@163.com
} 
function and adaptive bandwidth. Fansong Li et al. [7] proposed a modified KDE theory and application method based on the kernel density estimation theory, gave a KDE application method under various loading processes. Nonparametric rain flow extrapolation method is a method to estimate the unknown distribution parameters, it does not depend on the distribution model assumption, avoids the subjectivity in the process of extrapolation, this paper argues that nonparametric rain flow extrapolation is adaptable load extrapolation method on the load mileage extrapolation processing, using this way. At the same time, on the basis of mileage extrapolation, this paper proposes to combine mileage extrapolation and percentile extrapolation in order to reflect the difference in load characteristics.

\section{Frame load collection}

\subsection{Test principle}

In this paper, a strain test was used to collect the load time history of three different drivers in the ploughing condition of the dynamic mechanical vehicle frame, the strain condition of the structure was measured by pasting strain gauge, and then collected by data acquisition card. The schematic diagram is shown in figure 1 .

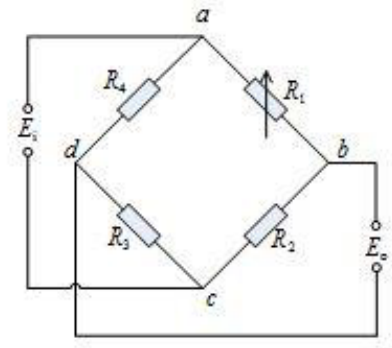

Figure 1. Schematic of wheatstone bridge test.

In the figure, $E_{i}$ is the excitation voltage of the bridge. $E_{o}$ is the bridge output voltage signal; $R_{1}$ is the measuring strain gauge, the rest is the fixed resistance.

$$
E_{o}=U_{a b}-U_{a d}=\frac{R_{1} R_{3}-R_{2} R_{4}}{\left(R_{1}+R_{2}\right)\left(R_{3}+R_{4}\right)} E_{i}
$$

When $R_{1}=R_{2}=R_{3}=R_{4}$, the bridge is a full-arm bridge and the output voltage $E_{o}=0$. When $R_{1}$ resistance changes $\Delta R$, bridge output voltage is:

$$
E_{o}=\frac{\Delta R}{4 R+2 \Delta R} E_{i}
$$

Because $\Delta R<<R$, ignore the item $2 \Delta R$, then :

$$
E_{o}=\frac{1}{4} \frac{\Delta R}{R} E_{i}=\frac{E_{i}}{4} K \varepsilon
$$

$\varepsilon$ is strain value, $K$ is the sensitivity coefficient of strain gauge, then:

$$
\varepsilon=\frac{4 E_{o}}{E_{i} K}
$$

Therefore, given the excitation voltage, sensitivity coefficient $K$, collecting the output signal $E_{o}$, the measured point strain data can be obtained.

\subsection{Selection of measuring points}


Firstly, the location of the measuring point is selected. In this paper, using the Workbench to do finite element analysis on frame, figure 2 is finite element model, and combined with the actual testing requirements, selecting 7 points as shown in figure 3 .

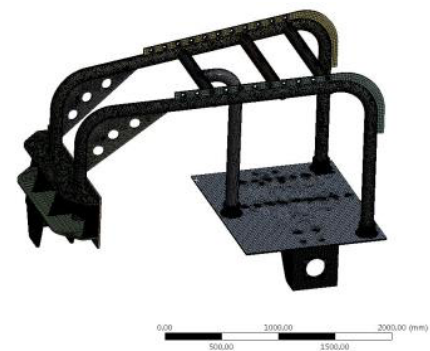

Figure 2. Finite element analysis model of frame.

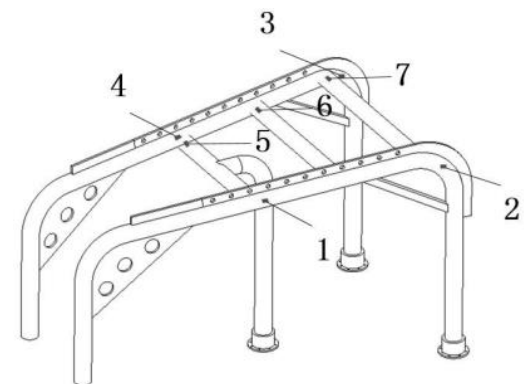

Figure 3. Measuring points.

\subsection{Test system construction}

Taking on load test in the working condition on the vegetable tool carrier driven by three different drivers. The strain testing system mainly includes BX120-3AA type single-axial strain gauge, and the sensitive grid resistance is $120 \Omega$, YSV7008 dynamic strain adjuster, YSV8016 network signal acquisition instrument, which consists of 16 data channels with a maximum sampling frequency of $16 \mathrm{KHz} /$ channel and an AD precision of 24 bits. The sampling frequency is $1024 \mathrm{~Hz}$, with a total of 7 data channels. The strain signal is collected and archived in ASCII data format, and each group is tested twice for comparison and analysis of data within the group.

\section{Signal processing and statistics}

\subsection{Signal processing}

Taking the first measuring point of ploughing condition as an example, the collected signal is filtered in nCode, the trend term is removed, and the singularity is eliminated [8], the comparison is shown in figure. 4.

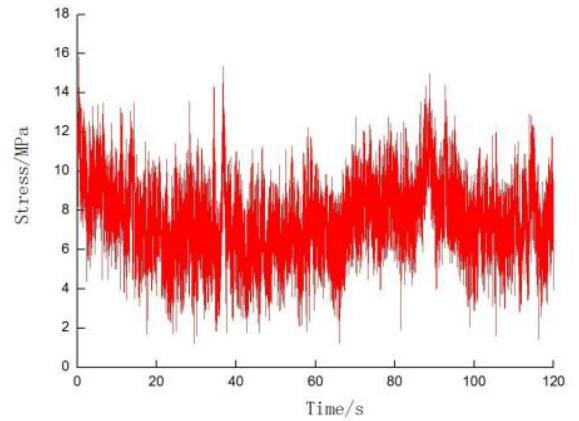

a. Unprocessed signal

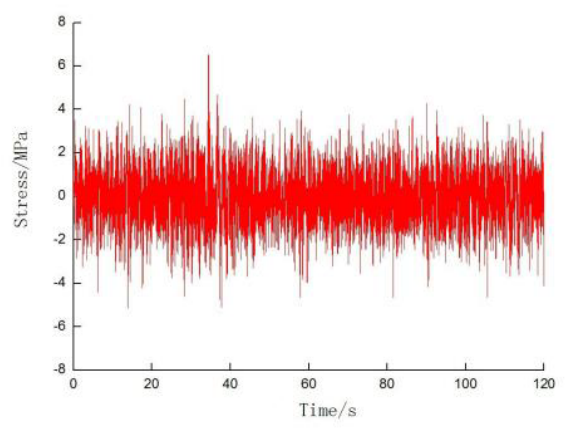

b. Processed signal

Figure 4. Signal of before and after processing.

\subsection{Signal statistics}

Mechanical components in practical engineering load is random, its characteristics is described by statistical method, the rain flow count method is the most widely used method in counting method [9], after counting post-processing by matrix and amplitude condition is shown in figure 5 . 


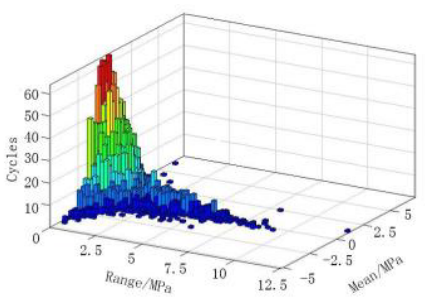

a. Load rainflow histogram

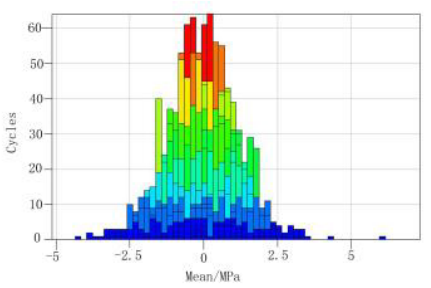

b. Load mean distribution

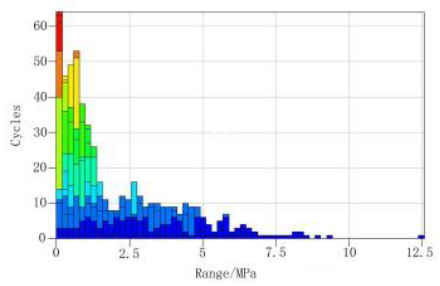

c. Load range distribution

Figure 5. Rain flow counting results of frame.

According to the amplitude probability distribution and test [10], the test results, determine the mean load obeys normal distribution, the load amplitude obeys weibull distribution, which is consistent with the characteristics of load data.

\section{Load mileage extrapolation}

\subsection{Kernel density estimation}

Based on the "From - to" rain flow matrix of non-parametric estimation method, under the premise that ensure that does not destroy the structure of the hysteresis loop back to load extrapolation, calculate the hysteresis loop back in the whole life cycle possible frequency [11]. $X_{1}, \ldots, X_{n}$ are sample of population, then the kernel density of the overall probability density function $f(x)$ at any point is estimated as:

$$
\overline{f_{h}}(x)=\frac{1}{n h} \sum_{i=1}^{n} K\left(\frac{x-X_{i}}{h}\right)
$$

$K\left(\frac{x-X_{i}}{h}\right)$ is kernel function, $h$ is the bandwidth, and the kernel function is required to satisfy:

$$
K(x) \geq 0, \int_{-\infty}^{+\infty} K(x) d x=1
$$

In kernel density estimation $\overline{f_{h}}(x)$, bandwidth $h$ determines the degree of smoothness, therefore, selection of proper bandwidth is particularly important in kernel density estimation, to select the appropriate bandwidth:

$$
M\left(\overline{f_{h}}\right)=E\left\{\int\left[\overline{f_{h}}(x)-f(x)\right]^{2} d x\right\}
$$

Where $f(x)$ is the overall true distribution density and $M$ is a function of bandwidth $h$ [12], the optimal bandwidth estimate can be obtained by finding its minimum point.

\subsection{Rain flow matrix processing and extrapolation}

The load data of the first measuring point is transformed into "From-to" rain flow matrix, and the mileage extrapolation of the test load is carried out, as shown in figure 6 . 


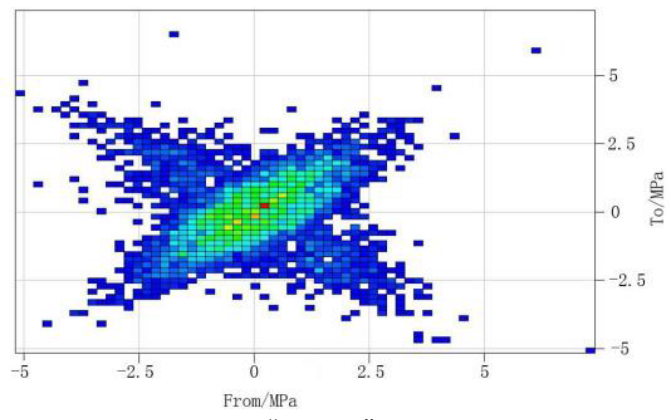

a. Original "From-to" matrix

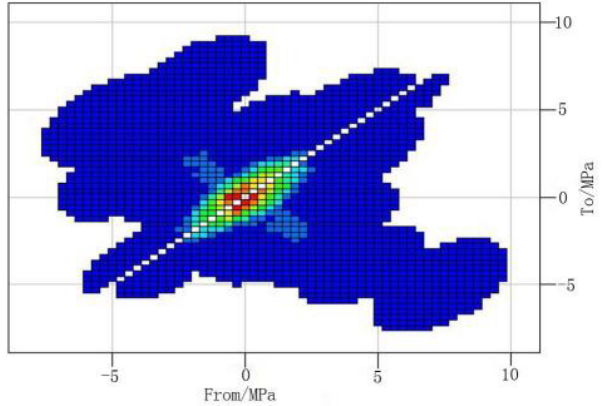

b. Mileage extrapolation matrix

Figure 6. Matrix of before and after mileage extrapolation.

\section{Load percentile extrapolation}

Collected vegetable tool carrier load of three different drivers, the load data of the three working conditions of mileage extrapolation were quantitatively extrapolated, taking first measuring point as an example. The results are shown in figure 7 :

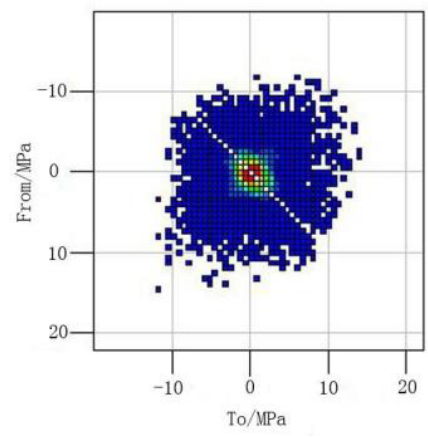

a. One condition

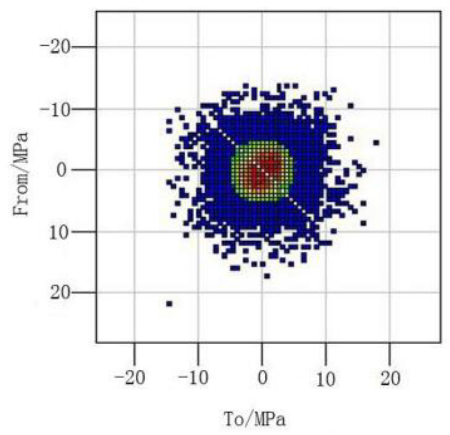

b. Three condition

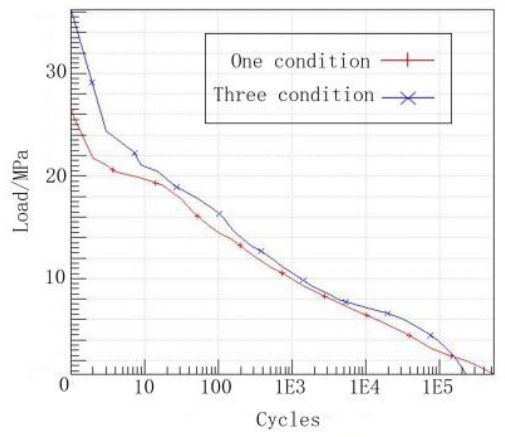

c. Comparision

Figure 7. Matrix of before and after percentile extrapolation.

\section{Frame load spectrum preparation}

In engineering practice, the non-uniform interval method is usually adopted for load classification [13], divided into 8 levels. Rain-flow statistics are carried out on the load data after mileage extrapolation and percentile extrapolation. The mean amplitude is divided into eight levels to form the eight-level two-dimensional load spectrum, as shown in the following table 1:

Table 1. Two-dimensional load spectrum.

\begin{tabular}{|c|c|c|c|c|c|c|c|c|}
\hline $\begin{array}{c}\text { Range } \\
\text { Mean } \\
\text { /MPa }\end{array}$ & 15.132 & 14.375 & 12.862 & 10.971 & 8.701 & 6.431 & 4.161 & 1.892 \\
\hline 6.566 & 0 & 0 & 0 & 0 & 0 & 0 & 3 & 111 \\
\hline 4.655 & 0 & 0 & 0 & 4 & 23 & 0 & 96 & 2612 \\
\hline 2.744 & 1 & 24 & 52 & 37 & 106 & 715 & 5224 & 83113 \\
\hline 0.833 & 0 & 6 & 19 & 296 & 2879 & 22200 & 49486 & 223724 \\
\hline-1.708 & 0 & 0 & 0 & 2 & 11 & 414 & 1432 & 46886 \\
\hline
\end{tabular}




\begin{tabular}{|c|c|c|c|c|c|c|c|c|}
\hline-2.989 & 0 & 0 & 0 & 0 & 0 & 0 & 10 & 1507 \\
\hline-4.900 & 0 & 0 & 0 & 0 & 0 & 0 & 0 & 2 \\
\hline-6.811 & 0 & 0 & 0 & 0 & 0 & 0 & 0 & 1 \\
\hline
\end{tabular}

\section{References}

1. Li Jianmin, Pei Zhongtao, Wen Buzheng, et al. An Analysis on the Load Spectrum and Fatigue Life of Loader's Driving Axle House[J]. Automotive Engeering, 2012, 34(11): 1020-1023+1032.

2. Lin Mingfang, Zhang Hongxin, Du Zhiqi, et al. Prediction of Fatigue life and Reliability Design in Automotive Semiaxle[J]. Automotive Engeering, 1991(04): 235-242.

3. Gao Yunkai, Xu Chengmin, Fang Jianguang. Study on the Programed Load Spectrum of the Body Fatigue Bench Test[J]. Journal of Mechanical Engeering, 2014, 50(04): 92-98.

4. Rodzewicz M. Determination and extrapolation of the glider load spectra[J]. Aircraft Engineering and Aerospace Technology, 2008, 487-496.

5. Socie D F, Pompetzki M A. Modeling Variability in Service Loading Spectrum[J]. Probabilistic Aspects of Life Predictions, ASTM STP, 2004, 1450: 46-57.

6. Drebler K, Gründer B, M. Hack et al. Extrapolation of rainflow matrices[J]. SAE Technical Paper, 1996.

7. Li Fansong, Wu Pingbo, Zengjing. Extrapolation of Load Histories Based on Kernel Density methods[J]. Journal of the China Railway Society, 2017, 39(07): 25-31.

8. Jia Haibo. Study on the Test and Generation Methods about Load Spectrum of Wheel Loader Driveline[D]. Changchun: Jilin University, 2009.

9. Liu Yongchen, Wang Guolin, Sun Li. Fatigue damage analysis and life prediction for vehicle control $\operatorname{arm}[\mathrm{J}]$. Transactions of the Chinese Society of Agricultural Engineering (Transactions of the CSAE), 2013, 29(16): 83-91.

10. Yan Chuliang. Investigation of compilation of fatigue load spectrum by two-parametric counting method[J]. Transactions of the Chinese Society for Agricultural Machinery, 1986(02): 94-102.

11. Gao Tianyu, Li Huanliang, Zheng Zheng, et al. Compiling method of load spectrum of ZLK50 type loader hydraulic cylinder based on rain flow frequency extrapolation[J]. Journal of Mechanical Strength, 2017, 39(04):951-956.

12. Silverman B. Density Estimation for Statistics and Data Analysis[M]. London: Chapman and Hall, 1986.

13. Zhai Xinting, Zhang Xiaochen, Jiang Zhujin, et al. Load spectrum compiling for wheel loader semi-axle based on mixed distribution[J]. Transactions of the Chinese Society of Agricultural Engineering (Transactions of the CSAE), 2018, 34(08): 78-84. 